\title{
The J-Generalized P - K Mittag-Leffler Function
}

\author{
Kuldeep Singh Gehlot and Anjana Bhandari
}

\author{
Government Bangur P.G. College Pali, \\ JNV University Jodhpur, Rajasthan, India-306401. \\ Email: drksgehlot@rediffmail.com \\ And \\ Department of Science and Humanities, \\ Swarrnim startup and Innovation University, \\ Gandhinagar, Gujarat, India- 382420. \\ Email: anjana.bhandari2011@gmail.com
}

\begin{abstract}
We know that the classical Mittag-Leffler function play an important role as solution of fractional order differential and integral equations. We introduce the j-generalized $\mathrm{p}-\mathrm{k}$ Mittag-leffler function. We evaluate the second order differential recurrence relation and four different integral representations and introduce a homogeneous linear differential equation whose one of the solution is the j-generalized p-k Mittag-Leffler function.

Also we evaluate the certain relations that exist between j-generalized $\mathrm{p}-\mathrm{k}$ Mittag-leffler function and Riemann-Liouville fractional integrals and derivatives.

We evaluate Mellin-Barnes integral representation of j-generalized p-k Mittag-Leffler Function. The relationship of j-generalized p-k Mittag-Leffler Function with Fox H-Function and Wright hypergeometric function is also establish. we obtained its Euler transform, Laplace Transform and Mellin transform.

Finally we derive some particular cases.
\end{abstract}

MSC(2011): 33E12, 33B10, 26A33.

Keywords: The j-generalized p - k Mittag-Leffler Function, The p - k Mittag-Leffler Function, Generalized k-Mittag-Leffler Function, Two parameter pochhammer symbol, Two parameter Gamma function.

\section{Introduction}

The two parameter pochhammer symbol is recently introduce by [9], equation 2.1, in the form,

\section{$1.1 \quad$ Definition}

Let $x \in C ; k, p \in R^{+}-\{0\}$ and $\operatorname{Re}(x)>0, n \in N$, the $\mathrm{p}$ - k Pochhammer Symbol (i.e. Two Parameter Pochhammer Symbol), ${ }_{p}(x)_{n, k}$ is given by

$$
{ }_{p}(x)_{n, k}=\left(\frac{x p}{k}\right)\left(\frac{x p}{k}+p\right)\left(\frac{x p}{k}+2 p\right) \ldots \ldots \ldots\left(\frac{x p}{k}+(n-1) p\right) .
$$

And the Two Parameter Gamma Function is given by [9], some of it's result are, 


\subsection{Definition}

For $x \in C / k Z^{-} ; k, p \in R^{+}-\{0\}$ and $\operatorname{Re}(x)>0, n \in N$, the $\mathrm{p}-\mathrm{k}$ Gamma Function (i.e. Two Parameter Gamma Function), ${ }_{p} \Gamma_{k}(x)$ as

$$
{ }_{p} \Gamma_{k}(x)=\frac{1}{k} \lim _{n \rightarrow \infty} \frac{n ! p^{n+1}(n p)^{\frac{x}{k}}}{p(x)_{n+1, k}} .
$$

or

$$
{ }_{p} \Gamma_{k}(x)=\frac{1}{k} \lim _{n \rightarrow \infty} \frac{n ! p^{n+1}(n p)^{\frac{x}{k}-1}}{{ }_{p}(x)_{n, k}} .
$$

The integral representation of $\mathrm{p}-\mathrm{k}$ Gamma Function is given by

$$
\begin{gathered}
{ }_{p} \Gamma_{k}(x)=\int_{0}^{\infty} e^{-\frac{t^{k}}{p}} t^{x-1} d t . \\
{ }_{p} \Gamma_{k}(x)=\left(\frac{p}{k}\right)^{\frac{x}{k}} \Gamma_{k}(x)=\frac{p^{\frac{x}{k}}}{k} \Gamma\left(\frac{x}{k}\right) . \\
{ }_{p}(x)_{n, k}=\left(\frac{p}{k}\right)^{n}(x)_{n, k}=(p)^{n}\left(\frac{x}{k}\right)_{n} .
\end{gathered}
$$

Also for Generalized p - k Pochhammer Symbol, we have

$$
\begin{gathered}
{ }_{p}(x)_{n q, k}=\left(\frac{p}{k}\right)^{n q}(x)_{n q, k}=(p)^{n q}\left(\frac{x}{k}\right)_{n q}=(p q)^{n q} \prod_{r=1}^{q}\left(\frac{\frac{x}{k}+r-1}{q}\right)_{n} . \\
{ }_{p}(x)_{n, k}=\frac{{ }_{p} \Gamma_{k}(x+n k)}{{ }_{p} \Gamma_{k}(x)} . \\
{ }_{p} \Gamma_{k}(x+k)=\frac{x p}{k}{ }_{p} \Gamma_{k}(x) . \\
n p_{p}(x)_{n-1, k}={ }_{p}(x)_{n, k}-{ }_{p}(x-k)_{n, k} .
\end{gathered}
$$

And

$$
{ }_{p}(x)_{n+j, k}={ }_{p}(x)_{j, k} \times{ }_{p}(x+j k)_{n, k} .
$$

The Mittag-Leffler function $E_{\alpha}(z)$ introduced by Gosta Mittag-Leffler [4] in 1903, defined as

$$
E_{\alpha}(z)=\sum_{n=0}^{\infty} \frac{z^{n}}{\Gamma(\alpha n+1)} .
$$

Here $z \in C, \alpha \geqslant 0$.

Wiman [2] generalized $E_{\alpha}(z)$ in 1905 and gave $E_{\alpha, \beta}(z)$ known as Wiman function, defined as

$$
E_{\alpha, \beta}(z)=\sum_{n=0}^{\infty} \frac{z^{n}}{\Gamma(\alpha n+\beta)} .
$$

Here $z, \alpha, \beta \in C ; \operatorname{Re}(\alpha)>0, \operatorname{Re}(\beta)>0$.

Prabhakar [17] in 1971, gave next generalization of Mittag-Leffler function and denoted as $E_{\alpha, \beta}^{\gamma}(z)$ and defined as

$$
E_{\alpha, \beta}^{\gamma}(z)=\sum_{n=0}^{\infty} \frac{(\gamma)_{n}}{\Gamma(\alpha n+\beta)} \frac{z^{n}}{n !} .
$$

Here $z, \alpha, \beta, \gamma \in C ; \operatorname{Re}(\alpha)>0, \operatorname{Re}(\beta)>0, \operatorname{Re}(\gamma)>0$. 
Shukla and Prajapati [1] in 2007, gave second generalization of Mittag-Leffler function and denoted it as $E_{\alpha, \beta}^{\gamma, q}(z)$ and defined as,

$$
E_{\alpha, \beta}^{\gamma, q}(z)=\sum_{n=0}^{\infty} \frac{(\gamma)_{n q}}{\Gamma(\alpha n+\beta)} \frac{z^{n}}{n !}
$$

Here $z, \alpha, \beta, \gamma \in C ; \operatorname{Re}(\alpha)>0, \operatorname{Re}(\beta)>0, \operatorname{Re}(\gamma)>0$ and $q \in(0,1) \cup N$.

The function $E_{\alpha, \beta}^{\gamma, q}(z)$ converges absolutely for all $z$ if $q<\operatorname{Re}(\alpha)+1$ and for $|z|<1$ if $q=\operatorname{Re}(\alpha)+1$. It is entire function of order $\frac{1}{\operatorname{Re}(\alpha)}$.

Gehlot K.S.[8] introduce Generalized k- Mittag-Leffler function in 2012, denoted as $G E_{k, \alpha, \beta}^{\gamma, q}(z)$ and defined for $k \in R ; z, \alpha, \beta, \gamma \in C ; \operatorname{Re}(\alpha)>0, \operatorname{Re}(\beta)>0, \operatorname{Re}(\gamma)>0$ and $q \in(0,1) \cup N$, as,

$$
G E_{k, \alpha, \beta}^{\gamma, q}(z)=\sum_{n=0}^{\infty} \frac{(\gamma)_{n q, k} z^{n}}{\Gamma_{k}(n \alpha+\beta)(n !)}
$$

where $(\gamma)_{n q, k}$ is the $\mathrm{k}$ - pochhammer symbol and $\Gamma_{k}(x)$ is the k-gamma function given by [15]. The generalized Pochhammer symbol is given as,

$$
(\gamma)_{n q}=\frac{\Gamma(\gamma+n q)}{\Gamma(\gamma)}=q^{q n} \prod_{r=1}^{q}\left(\frac{\gamma+r-1}{q}\right)_{n}, \text { if } q \in N
$$

Gehlot K.S.[9], Introduce The p- k Mittag-Leffler function in 2018, denoted by ${ }_{p} E_{k, \alpha, \beta}^{\gamma, q}(z)$ and defined for $k, p \in R^{+}-\{0\} ; \alpha, \beta, \gamma \in C / k Z^{-} ; \operatorname{Re}(\alpha)>0, \operatorname{Re}(\beta)>0, \operatorname{Re}(\gamma)>0$ and $q \in(0,1) \cup N$.

$$
{ }_{p} E_{k, \alpha, \beta}^{\gamma, q}(z)=\sum_{n=0}^{\infty} \frac{p(\gamma)_{n q, k}}{{ }_{p} \Gamma_{k}(n \alpha+\beta)} \frac{z^{n}}{n !} .
$$

Where ${ }_{p}(\gamma)_{n q, k}$ is two parameter Pochhammer symbol given by equation (1.1) and ${ }_{p} \Gamma_{k}(x)$ is the two parameter Gamma function given by equation (1.3).

L.L.Luque [14]in the year 2019, introduce the L-mittag-Leffler function defined for $\alpha, \beta, \gamma \in$ $C ; \operatorname{Re}(\alpha)>0, \operatorname{Re}(\beta)>0, \operatorname{Re}(\gamma)>0, j \in N_{0}$ by the series

$$
L_{\alpha, \beta}^{\gamma, j}(z)=\sum_{n=0}^{\infty} \frac{(\gamma)_{n+j}}{\Gamma(n \alpha+\beta)} \frac{z^{n}}{(n+j) !}, \quad(z \in C) .
$$

The Fractional Integral operators ([16], Definition 2.1, Page 33) are defined as,

$$
\left(I_{0+}^{\vartheta} f\right)(z)=\frac{1}{\Gamma(\vartheta)} \int_{0}^{z} \frac{f(t)}{(z-t)^{1-\vartheta}} d t,(z>0)
$$

and

$$
\left(I_{-}^{\vartheta} f\right)(z)=\frac{1}{\Gamma(\vartheta)} \int_{z}^{\infty} \frac{f(t)}{(t-z)^{1-\vartheta}} d t,(z>0),
$$

The Fractional Derivative ([16], Definition 2.2, Page 35) are defined as,

$$
\begin{gathered}
\left(D_{0+}^{\vartheta} f\right)(z)=\left(\frac{d}{d z}\right)^{[\operatorname{Re}(\vartheta)]+1}\left(I_{0+}^{1-\vartheta+[\operatorname{Re}(\vartheta)]} f\right)(z) \\
=\frac{1}{\Gamma(1-\vartheta+[\operatorname{Re}(\vartheta)])}\left(\frac{d}{d z}\right)^{[\operatorname{Re}(\vartheta)]+1} \int_{0}^{z} \frac{f(t)}{(z-t)^{\vartheta-[\operatorname{Re}(\vartheta)]}} d t,(z>0),
\end{gathered}
$$

and

$$
\left(D_{-}^{\vartheta} f\right)(z)=\left(-\frac{d}{d z}\right)^{[\operatorname{Re}(\vartheta)]+1}\left(I_{-}^{1-\vartheta+[\operatorname{Re}(\vartheta)]} f\right)(z)
$$




$$
=\frac{1}{\Gamma(1-\vartheta+[\operatorname{Re}(\vartheta)])}\left(-\frac{d}{d z}\right)^{[\operatorname{Re}(\vartheta)]+1} \int_{z}^{\infty} \frac{f(t)}{(t-z)^{\vartheta-[\operatorname{Re}(\vartheta)]}} d t,(z>0) .
$$

Where $\vartheta \in C(\operatorname{Re}(\vartheta)>0)$.

Wright generalized hypergeometric function [15];

$$
\begin{gathered}
{ }_{p} \psi_{q}\left[\begin{array}{c}
\left(\alpha_{1}, A_{1}\right), \ldots,\left(\alpha_{p}, A_{p}\right) ; z \\
\left(\beta_{1}, B_{1}\right), \ldots,\left(\beta_{q}, B_{q}\right) ;
\end{array}\right]=\sum_{n=0}^{\infty} \frac{\prod_{i=1}^{p} \Gamma\left(\alpha_{i}+A_{i} n\right) z^{n}}{\prod_{j=1}^{q} \Gamma\left(\beta_{i}+B_{i} n\right) n !} . \\
{ }_{p} \psi_{q}\left[\begin{array}{c}
\left(\alpha_{1}, A_{1}\right), \ldots,\left(\alpha_{p}, A_{p}\right) ; \\
\left(\beta_{1}, B_{1}\right), \ldots,\left(\beta_{q}, B_{q}\right) ;
\end{array}\right]=H_{p, q+1}^{1, p}\left[\begin{array}{r}
\left.-\alpha_{1}, A_{1}\right), \ldots,\left(1-\alpha_{p}, A_{p}\right) ; \\
(0,1),\left(1-\beta_{1}, B_{1}\right), \ldots,\left(1-\beta_{q}, B_{q}\right) ;
\end{array}\right] .
\end{gathered}
$$

Where $H_{p, q}^{m, n}[$.$] denotes the Fox H-function.$

Euler Beta transform, [6],

$$
B[f(z): a, b]=\int_{0}^{1} z^{a-1}(1-z)^{b-1} f(z) d z .
$$

Laplace transform,([7],equation3.1.1),

$$
L[f(z): s]=\int_{0}^{\infty} e^{-s z} f(z) d z .
$$

Mellin transform,([7],equation 4.1.1),

$$
M[f(z): s]=\int_{0}^{\infty} z^{s-1} f(z) d z=f^{*}(s), \operatorname{Re}(s)>0,
$$

then

$$
f(z)=M^{-1}\left[f^{*}(s): x\right]=\int f^{*}(s) x^{-s} d s
$$

Throughout this paper Let $C, R^{+}, R e(), Z^{-}, N_{0}$ and $N$ be the sets of complex numbers, positive real numbers, real part of complex number, negative integer, whole number and natural numbers respectively.

\section{The j-generelized $\mathrm{p}$ - k Mittag-Leffler function}

In this section we introduce the $\mathrm{j}$-generelized $\mathrm{p}-\mathrm{k}$ Mittag-Leffler function and prove some of its properties.

\subsection{Definition}

Let $k, p \in R^{+}-\{0\} ; \alpha, \beta, \gamma \in C / k Z^{-} ; \operatorname{Re}(\alpha)>0, \operatorname{Re}(\beta)>0, \operatorname{Re}(\gamma)>0, j \in N_{0}$ and $q \in$ $(0,1) \cup N$. The j-generelized p - k Mittag-Leffler function denoted by ${ }_{p}^{j} E_{k, \alpha, \beta}^{\gamma, q}(z)$ and defined as

$$
{ }_{p}^{j} E_{k, \alpha, \beta}^{\gamma, q}(z)=\sum_{n=0}^{\infty} \frac{p(\gamma)_{(n+j) q, k}}{{ }_{p} \Gamma_{k}(n \alpha+\beta)} \frac{z^{n}}{(n+j) !}, \quad z \in C .
$$

Where ${ }_{p}(\gamma)_{n q, k}$ is two parameter Pochhammer symbol given by equation $(1.1)$ and ${ }_{p} \Gamma_{k}(x)$ is the two parameter Gamma function given by equation (1.3). 
Particular cases : For some particular values of the parameters $j, p, q, k, \alpha, \beta, \gamma$ we can obtain certain defined and undefined Mittag-Leffler functions:

(a) For $j=0$ equation (2.1), reduces in the p-k Mittag-Leffler functions defined by [10],

$$
{ }_{p}^{0} E_{k, \alpha, \beta}^{\gamma, q}(z)=\sum_{n=0}^{\infty} \frac{p(\gamma)_{n q, k}}{{ }_{p} \Gamma_{k}(n \alpha+\beta)} \frac{z^{n}}{n !}, \quad z \in C .
$$

(b) For $q=1$ equation (2.1), reduces in $\mathrm{j}$ form of $\mathrm{p}-\mathrm{k}$ Mittag-Leffler functions defined as,

$$
{ }_{p}^{j} E_{k, \alpha, \beta}^{\gamma, 1}(z)=\sum_{n=0}^{\infty} \frac{p(\gamma)_{(n+j), k}}{{ }_{p} \Gamma_{k}(n \alpha+\beta)} \frac{z^{n}}{(n+j) !}, \quad z \in C .
$$

(c) For $q=1, p=k$ equation (2.1), reduces in $\mathrm{j}$ form of $\mathrm{k}$ - Mittag-Leffler functions defined as,

$$
{ }_{k}^{j} E_{k, \alpha, \beta}^{\gamma, 1}(z)=\sum_{n=0}^{\infty} \frac{(\gamma)_{(n+j), k}}{\Gamma_{k}(n \alpha+\beta)} \frac{z^{n}}{(n+j) !}, \quad z \in C .
$$

(d) For $q=1, j=0$ equation (2.1), reduces in generalized form of k- Mittag-Leffler functions defined as.

$$
{ }_{p} E_{k, \alpha, \beta}^{\gamma, 1}(z)=\sum_{n=0}^{\infty} \frac{p(\gamma)_{n, k} z^{n}}{{ }_{p} \Gamma_{k}(n \alpha+\beta)(n !)} .
$$

(e) For $p=k, j=0$ equation (2.1), reduces in Generalized k- Mittag-Leffler functions defined by $[8]$.

$$
{ }_{k} E_{k, \alpha, \beta}^{\gamma, q}(z)=\sum_{n=0}^{\infty} \frac{{ }_{k}(\gamma)_{n q, k} z^{n}}{{ }_{k}(n \alpha+\beta)(n !)}=G E_{k, \alpha, \beta}^{\gamma, q}(z) .
$$

(f) For $p=k, q=1, j=0$ equation (2.1), reduces in $\mathrm{k}$ - Mittag-Leffler functions defined by [3].

$$
{ }_{k} E_{k, \alpha, \beta}^{\gamma, 1}(z)=\sum_{n=0}^{\infty} \frac{(\gamma)_{n, k} z^{n}}{\Gamma_{k}(n \alpha+\beta)(n !)}=E_{k, \alpha, \beta}^{\gamma}(z) .
$$

(g) For $p=k$ and $k=1, j=0$ equation (2.1), reduces in Mittag-Leffler functions defined by [1].

$$
{ }_{1} E_{1, \alpha, \beta}^{\gamma, q}(z)=\sum_{n=0}^{\infty} \frac{(\gamma)_{n q} z^{n}}{\Gamma(n \alpha+\beta)(n !)}=E_{\alpha, \beta}^{\gamma, q}(z) .
$$

(h)For $p=k=q=1$ equation (2.1), reduces in L-Mittag-Leffler functions defined by [14].

$$
{ }_{1} E_{1, \alpha, \beta}^{\gamma, 1}(z)=\sum_{n=0}^{\infty} \frac{(\gamma)_{n+j} z^{n}}{\Gamma(n \alpha+\beta)(n+j) !}=L_{\alpha, \beta}^{\gamma, j}(z) .
$$

(i) For $p=k, q=1, j=0$ and $k=1$ equation (2.1), reduces in Mittag-Leffler functions defined by [17].

$$
{ }_{1} E_{1, \alpha, \beta}^{\gamma, 1}(z)=\sum_{n=0}^{\infty} \frac{(\gamma)_{n} z^{n}}{\Gamma(n \alpha+\beta)(n !)}=E_{\alpha, \beta}^{\gamma}(z),
$$

(j) For $p=k, q=1, k=1, j=0$ and $\gamma=1$ equation (2.1), reduces in Mittag-Leffler functions defined by [3].

$$
{ }_{1} E_{1, \alpha, \beta}^{1,1}(z)=\sum_{n=0}^{\infty} \frac{z^{n}}{\Gamma(n \alpha+\beta)}=E_{\alpha, \beta}(z),
$$


(k) For $p=k, q=1, k=1, \gamma=1, j=0$ and $\beta=1$ equation (2.1), reduces in Mittag-Leffler functions defined by [4].

$$
{ }_{1} E_{1, \alpha, 1}^{1,1}(z)=\sum_{n=0}^{\infty} \frac{z^{n}}{\Gamma(n \alpha+1)}=E_{\alpha}(z)
$$

Theorem 2.1: The j-generelized p - k Mittag-Leffler function defined by equation (2.1) is an entire function of order

$$
\frac{1}{\rho}=\operatorname{Re}\left(\frac{\alpha}{k}\right)-q+1
$$

Proof: Let $\mathrm{R}$ is the radius of convergence of the j-generelized $\mathrm{p}-\mathrm{k}$ Mittag-Leffler function. The asymptotic Striling formula for Gamma function and factorial are given by, [5]

$$
\Gamma(a z+b)=\sqrt{2 \pi} e^{-a z}(a z)^{a z+b-\frac{1}{2}}\left[1+o\left(\frac{1}{z}\right)\right],(\arg (a z+b)<\pi ;|z| \rightarrow \infty) .
$$

and

$$
n !=\sqrt{2 \pi} e^{-n}(n)^{n+\frac{1}{2}}\left[1+o\left(\frac{1}{n}\right)\right],(n \in N ; n \rightarrow \infty) .
$$

From equation (2.1), we have

$$
{ }_{p}^{j} E_{k, \alpha, \beta}^{\gamma, q}(z)=\sum_{n=0}^{\infty} \frac{p(\gamma)_{(n+j) q, k}}{{ }_{p}(n \alpha+\beta)} \frac{z^{n}}{(n+j) !}=\sum_{n=0}^{\infty} C_{n} z^{n},
$$

since

$$
\begin{aligned}
& R=\limsup _{n \rightarrow \infty}\left|\frac{C_{n}}{C_{n+1}}\right|, \\
&\left|\frac{C_{n}}{C_{n+1}}\right|=\left|\frac{p(\gamma)_{(n+j) q, k}}{{ }_{p} \Gamma_{k}(n \alpha+\beta)} \frac{1}{(n+j) !} \times \frac{{ } \Gamma_{k}(n \alpha+\alpha+\beta)(n+1+j) !}{p(\gamma)_{(n+1+j) q, k}}\right|
\end{aligned}
$$

using equations (2.19) and (2.20) of [9], we have

$$
\left|\frac{C_{n}}{C_{n+1}}\right|=(n+1+j)\left|p^{\frac{\alpha-q k}{k}}\right|\left|\frac{\Gamma\left(n q+j q+\frac{\gamma}{k}\right)}{\Gamma\left(n q+j q+q+\frac{\gamma}{k}\right)}\right|\left|\frac{\Gamma\left(\frac{n \alpha+\alpha+\beta}{k}\right)}{\Gamma\left(\frac{n \alpha+\beta}{k}\right)}\right|,
$$

using equation (2.15), we have

$$
\simeq\left|p^{\frac{\alpha}{k}-q}\right|\left|q^{-q}\right|\left|\left(\frac{\alpha}{k}\right)^{\frac{\alpha}{k}}\right|\left|n^{\frac{\alpha}{k}+1-q}\right| \rightarrow \infty
$$

when,

$$
\operatorname{Re}\left(\frac{\alpha}{k}+1-q\right)>0,
$$

Thus, the j-generelized $\mathrm{p}-\mathrm{k}$ Mittag-Leffler function is an entire function for $q<\operatorname{Re}\left(\frac{\alpha}{k}\right)+1$ To determine the order $\rho$,

$$
\begin{gathered}
\rho=\limsup _{n \rightarrow \infty} \frac{n \ln n}{\ln \left(\frac{1}{\left|C_{n}\right|}\right)}, \\
\left|\frac{1}{C_{n}}\right|=\left|\frac{{ }_{p} \Gamma_{k}(n \alpha+\beta)(n+j) !}{p(\gamma)_{(n+j) q, k}}\right|,
\end{gathered}
$$

using theorem 2.19 and 2.20 of [9], we have

$$
\left|\frac{1}{C_{n}}\right|=\frac{(n+j) !}{k}\left|p^{\frac{\gamma}{k}+\frac{n \alpha+\beta}{k}-\frac{\gamma+(n+j) q k}{k}}\right|\left|\frac{\Gamma\left(\frac{\gamma}{k}\right) \Gamma\left(\frac{n \alpha+\beta}{k}\right)}{\Gamma\left(\frac{\gamma}{k}+(n+j) q\right)}\right|,
$$


By using equation (2.12) and (2.13), we get

$$
\left|\frac{1}{C_{n}}\right|=k^{-1}(2 \pi)^{\frac{1}{2}}\left|p^{\left(\frac{\alpha-q k}{k}\right) n+\frac{\beta}{k}-j q}\right|\left|\left(\frac{\alpha}{k}\right)^{\frac{n \alpha}{k}+\frac{\beta}{k}-\frac{1}{2}}\right|\left|n^{\frac{n \alpha}{k}+\frac{\beta}{k}-\frac{\gamma}{k}-n q-j q+n+j+\frac{1}{2}}\right|\left|e^{-n \operatorname{Re}\left(\frac{\alpha}{k}+1-q\right)}\right|
$$

taking $l n$ of above equation and put in equation (2.13), we have the order of j-generelized $\mathrm{p}-\mathrm{k}$ Mittag-Leffler function is given by

$$
\rho=\frac{k}{R e(\alpha)-q k+k}
$$

Hence.

Theorem 2.2: The functional relation between the j-generelized $\mathrm{p}-\mathrm{k}$ Mittag-Leffler function given by equation (2.1) with $\mathrm{p}-\mathrm{k}$ Mittag-Leffler function defined by [10] and generelized Mittag-Leffler function defined by [1] are given by

$$
\begin{gathered}
{ }_{p}^{j} E_{k, \alpha, \beta}^{\gamma, q}(z)=\left(k p^{j q-\frac{\beta}{k}}\right){ }^{j} E_{\frac{\alpha}{k}, \frac{\beta}{k}}^{\frac{\gamma}{k}, q}\left(z p^{q-\frac{\alpha}{k}}\right) . \\
\left(\frac{d}{d z}\right)^{l}\left[z^{j} \times{ }_{p}^{j} E_{k, \alpha, \beta}^{\gamma, q}(z)\right]={ }_{p}(\gamma)_{l q, k} z^{j-l}{ }_{p}^{j-l} E_{k, \alpha, \beta}^{\gamma+l q k, q}(z), \text { for } l<j . \\
\left(\frac{d}{d z}\right)^{l}\left[z^{j} \times{ }_{p}^{j} E_{k, \alpha, \beta}^{\gamma, q}(z)\right]={ }_{p}(\gamma)_{l q, k}{ }_{p} E_{k, \alpha, \beta}^{\gamma+l q k, q}(z), \text { for } l=j . \\
\left(\frac{d}{d z}\right)^{l}\left[z^{j} \times{ }_{p}^{j} E_{k, \alpha, \beta}^{\gamma, q}(z)\right]={ }_{p}(\gamma)_{l q, k}{ }_{p} E_{k, \alpha, \beta+l \alpha-j \alpha}^{\gamma+l q k, q}(z), \text { for } l>j .
\end{gathered}
$$

\section{Proof of equation (2.16)}

Using equation (1.5) and (1.6), we get the desired result.

Proof of equation (2.17), (2.18) and (2.19)

Using the equation (2.1), in right hand side of (2.17), we have

$$
\frac{d^{l}}{d z^{l}}\left[z^{j} \times{ }_{p}^{j} E_{k, \alpha, \beta}^{\gamma, q}(z)\right]=\sum_{n=0}^{\infty} \frac{p(\gamma)_{(n+j) q, k}}{{ }_{p} \Gamma_{k}(n \alpha+\beta)} \frac{z^{n+j-l}}{(n+j-l) !},
$$

using equation (1.11), we have

$$
\frac{d^{l}}{d z^{l}}\left[z^{j} \times{ }_{p}^{j} E_{k, \alpha, \beta}^{\gamma, q}(z)\right]=\sum_{n=0}^{\infty} \frac{{ }_{p}(\gamma)_{l q, k p}(\gamma+l q k)_{(n+j-l) q, k}}{{ }_{p} \Gamma_{k}(n \alpha+\beta)} \frac{z^{n+j-l}}{(n+j-l) !},
$$

hence we have,

$$
\begin{gathered}
={ }_{p}(\gamma)_{l q, k} \quad z^{j-l}{ }_{p}^{j-l} E_{k, \alpha, \beta}^{\gamma+l q k, q}(z), \text { for } l<j . \\
={ }_{p}(\gamma)_{j q, k}{ }_{p} E_{k, \alpha, \beta}^{\gamma+l q k,}(z), \text { for } l=j . \\
={ }_{p}(\gamma)_{l q, k}{ }_{p} E_{k, \alpha, \beta+l \alpha-j \alpha}^{\gamma+l q k, q}(z), \text { for } l>j .
\end{gathered}
$$

Theorem 2.3: The following elementary properties are satisfied by the j-generelized $\mathrm{p}-\mathrm{k}$ Mittag-Leffler function defined by equation (2.1),

$$
\begin{gathered}
k{ }_{p}^{j} E_{k, \alpha, \beta}^{\gamma, q}(z)=p \beta{ }_{p}^{j} E_{k, \alpha, \beta+k}^{\gamma, q}(z)+z p \alpha \frac{d}{d z}{ }_{p}^{j} E_{k, \alpha, \beta+k}^{\gamma, q}(z) . \\
p q p(\gamma)_{q-1, k}{ }_{p}^{j-1} E_{k, \alpha, \beta}^{\gamma+k q-k, q}(z)={ }_{p}^{j} E_{k, \alpha, \beta}^{\gamma, q}(z)-{ }_{p}^{j} E_{k, \alpha, \beta}^{\gamma-k, q}(z) . \\
\sum_{n=0}^{\infty}(x+y)^{n}{ }_{p}^{j} E_{k, 0, n k+j k+k}^{n q k+k, q}(x y)=\sum_{r=0}^{\infty} \frac{{ }_{p} \Gamma_{k}(r q k+k)(x y p)^{r}}{{ }_{p} \Gamma_{k}(r k+j k+k)} \times{ }_{p}^{j} E_{k, q k, k}^{r q k+k, q}\left(\frac{x+y}{p}\right) .
\end{gathered}
$$




\section{Proof of equation (2.20)}

Consider the right hand side of equation (2.20),

$$
A \equiv p \beta{ }_{p}^{j} E_{k, \alpha, \beta+k}^{\gamma, q}(z)+z p \alpha \frac{d}{d z}{ }_{p}^{j} E_{k, \alpha, \beta+k}^{\gamma, q}(z),
$$

using equation (2.1),

$$
\begin{gathered}
A \equiv p \beta \sum_{n=0}^{\infty} \frac{p(\gamma)_{(n+j) q, k}}{{ }_{p} \Gamma_{k}(n \alpha+\beta+k)} \frac{z^{n}}{(n+j) !}+z p \alpha \sum_{n=0}^{\infty} \frac{p(\gamma)_{(n+j) q, k}}{{ }_{p} \Gamma_{k}(n \alpha+\beta+k)} \frac{n z^{n-1}}{(n+j) !}, \\
A \equiv p \sum_{n=0}^{\infty} \frac{{ }_{p}(\gamma)_{(n+j) q, k}(n \alpha+\beta)}{{ }_{p} \Gamma_{k}(n \alpha+\beta+k)} \frac{z^{n}}{(n+j) !},
\end{gathered}
$$

using the equation (1.9), we have

$$
A \equiv k{ }_{p}^{j} E_{k, \alpha, \beta}^{\gamma, q}(z)
$$

Proof of equation (2.21)

Consider the right hand side of (2.21),

$$
A \equiv{ }_{p}^{j} E_{k, \alpha, \beta}^{\gamma, q}(z)-{ }_{p}^{j} E_{k, \alpha, \beta}^{\gamma-k, q}(z),
$$

using equation (2.1), we have

$$
A \equiv \sum_{n=0}^{\infty} \frac{z^{n}}{{ }_{p} \Gamma_{k}(n \alpha+\beta)(n+j) !}\left[p(\gamma)_{(n+j) q, k}-{ }_{p}(\gamma-k)_{(n+j) q, k}\right],
$$

using equations (1.10) and (1.11), we have

$$
A \equiv p q_{p}(\gamma)_{q-1, k}{ }_{p}^{j-1} E_{k, \alpha, \beta}^{\gamma+k q-k, q}(z) .
$$

Proof of equation (2.22)

Consider the Left hand side of equation (2.22),

$$
A \equiv \sum_{n=0}^{\infty}(x+y)^{n}{ }_{p}^{j} E_{k, 0,(n+j+1) k}^{n q k+k, q}(x y),
$$

using equation (2.1), we have

$$
A \equiv \sum_{n=0}^{\infty}(x+y)^{n} \sum_{r=0}^{\infty} \frac{{ }_{p}(n q k+k)_{(r+j) q, k}}{{ }_{p} \Gamma_{k}(n k+j k+k)} \frac{(x y)^{r}}{(r+j) !},
$$

now simplifying, by using equation (1.5) and (1.6), we have

$$
\begin{aligned}
& { }_{p}(n q k+k)_{(r+j) q, k}=p^{(r+j) q}(n q+1)_{(r+j) q}, \\
& =p^{(r+j) q} \frac{\Gamma(n q+(r+j) q+1)}{\Gamma(n q+1))}, \\
& =p^{(r+j) q} \frac{\Gamma((r+j) q+1+n q)}{\Gamma((r+j) q+1)} \frac{\Gamma((r+j) q+1)}{\Gamma(n q+1)}, \\
& ={ }_{p} \Gamma_{k}(r q k+k) \frac{p(r q k+k)_{(r+j) q, k}}{{ }_{p} \Gamma_{k}(n q k+k)},
\end{aligned}
$$

then equation (2.23) becomes by rearranging the terms, we have

$$
A \equiv \sum_{r=0}^{\infty} \frac{{ }_{p} \Gamma_{k}(r q k+k)(x y p)^{r}}{{ }_{p} \Gamma_{k}(r k+j k+k)} \sum_{n=0}^{\infty} \frac{p(r q k+k)_{(n+j) q, k}}{{ }_{p} \Gamma_{k}(q k n+k)(n+j) !}\left(\frac{x+y}{p}\right)^{n} .
$$

This completes the proof. 


\section{Fractional Integral and Differentiation of the j-generalized p - k Mittag-Leffler Function}

In this section we evaluate certain relations that exist between the j-generalized $\mathrm{p}-\mathrm{k}$ MittagLeffler Function and Riemann-Liouville fractional integrals and derivatives. It has been shown that the fractional integration and differentiation operators of j-generalized $\mathrm{p}-\mathrm{k}$ Mittag-Leffler Function with power multipliers into the function of the same form. Also point out some special cases.

Theorem 3.1 The left-side Riemann-Liouville Fractional Integral Operator $I_{0^{+}}^{\vartheta}$ of the j-generalized $\mathrm{p}$ - k Mittag-Leffler Function is given by,

$$
\left(I_{0^{+}}^{\vartheta}\left[t^{\frac{\beta}{k}-1}{ }_{p}^{j} E_{k, \alpha, \beta}^{\gamma, q}\left(t^{\frac{\alpha}{k}}\right)\right]\right)(z)=p^{\vartheta} z^{\frac{\beta}{k}+\vartheta-1}{ }_{p}^{j} E_{k, \alpha, \beta+k \vartheta}^{\gamma, q}\left(z^{\frac{\alpha}{k}}\right) .
$$

Where

$k, p \in R^{+}-\{0\} ; \alpha, \beta, \gamma \in C / k Z^{-} ; \operatorname{Re}(\alpha)>0, \operatorname{Re}(\beta)>0, \operatorname{Re}(\gamma)>0, j \in N_{0}, q \in(0,1) \cup N$ and $\operatorname{Re}(\vartheta)>0$.

Proof: Consider left hand side,

$$
A \equiv\left(I_{0^{+}}^{\vartheta}\left[t^{\frac{\beta}{k}-1}{ }_{p}^{j} E_{k, \alpha, \beta}^{\gamma, q}\left(t^{\frac{\alpha}{k}}\right)\right]\right)(z),
$$

by virtue of equation (1.20) and (2.1), we have

$$
A \equiv \frac{1}{\Gamma(\vartheta)} \int_{0}^{z} \frac{t^{\frac{\beta}{k}-1}}{(z-t)^{1-\vartheta}} \sum_{n=0}^{\infty} \frac{{ }_{p}(\gamma)_{(n+j) q, k}}{\Gamma_{k}(n \alpha+\beta)} \frac{t^{\frac{n \alpha}{k}}}{(n+j)} d t,
$$

interchanging the order of integration and summation and evaluate the inner integral by substitute $t=z u$ and using the beta function formula, it gives

$$
A \equiv \frac{z^{\frac{\beta}{k}+\vartheta-1}}{\Gamma(\vartheta)} \sum_{n=0}^{\infty} \frac{p(\gamma)_{(n+j) q, k}}{{ }_{p} \Gamma_{k}(n \alpha+\beta)} \frac{z^{\frac{n \alpha}{k}}}{(n+j) !} \frac{\Gamma\left(\frac{n \alpha+\beta}{k}\right) \Gamma(\vartheta)}{\Gamma\left(\frac{n \alpha+\beta+k \vartheta}{k}\right)},
$$

using the equation (1.5), we have

$$
A \equiv p^{\vartheta} z^{\frac{\beta}{k}+\vartheta-1}{ }_{p}^{j} E_{k, \alpha, \beta+k \vartheta}^{\gamma, q}\left(z^{\frac{\alpha}{k}}\right)
$$

Hence, we get the desired result.

Theorem 3.2 The right-side Riemann-Liouville Fractional Integral Operator $I_{-}^{\vartheta}$ of the j-generalized $\mathrm{p}$ - k Mittag-Leffler Function is given by,

$$
\left(I_{-}^{\vartheta}\left[t^{-\frac{\beta}{k}-\vartheta}{ }_{p}^{j} E_{k, \alpha, \beta}^{\gamma, q}\left(t^{-\frac{\alpha}{k}}\right)\right]\right)(z)=p^{\vartheta} z^{-\frac{\beta}{k}}{ }_{p}^{j} E_{k, \alpha, \beta+k \vartheta}^{\gamma, q}\left(z^{-\frac{\alpha}{k}}\right) .
$$

Where

$$
k, p \in R^{+}-\{0\} ; \alpha, \beta, \gamma \in C / k Z^{-} ; \operatorname{Re}(\alpha)>0, \operatorname{Re}(\beta)>0, \operatorname{Re}(\gamma)>0, j \in N_{0}, q \in(0,1) \cup N \text { and } \operatorname{Re}(\vartheta)>0 .
$$

Proof: Consider left hand side,

$$
A \equiv\left(I_{-}^{\vartheta}\left[t^{-\frac{\beta}{k}-\vartheta}{ }_{p}^{j} E_{k, \alpha, \beta}^{\gamma, q}\left(t^{-\frac{\alpha}{k}}\right)\right]\right)(z)
$$


by virtue of equation (1.21) and (2.1), we have

$$
A \equiv \frac{1}{\Gamma(\vartheta)} \int_{z}^{\infty} \frac{t^{-\frac{\beta}{k}-\vartheta}}{(t-z)^{1-\vartheta}} \sum_{n=0}^{\infty} \frac{p(\gamma)_{(n+j) q, k}}{{ }_{p} \Gamma_{k}(n \alpha+\beta)} \frac{t^{-\frac{n \alpha}{k}}}{(n+j) !} d t
$$

interchanging the order of integration and summation and evaluate the inner integral by substitute $t=\frac{z}{u}$ and using the beta function formula, it gives

$$
A \equiv \frac{z^{-\frac{\beta}{k}}}{\Gamma(\vartheta)} \sum_{n=0}^{\infty} \frac{p(\gamma)_{(n+j) q, k}}{{ }_{p} \Gamma_{k}(n \alpha+\beta)} \frac{z^{-\frac{n \alpha}{k}}}{(n+j) !} \frac{\Gamma\left(\frac{n \alpha+\beta}{k}\right) \Gamma(\vartheta)}{\Gamma\left(\frac{n \alpha+\beta+k \vartheta}{k}\right)},
$$

using the equation (1.5), we have

$$
A \equiv p^{\vartheta} z^{-\frac{\beta}{k}}{ }_{p}^{j} E_{k, \alpha, \beta+k \vartheta}^{\gamma, q}\left(z^{-\frac{\alpha}{k}}\right)
$$

Hence, we get the desired result.

Theorem 3.3 The left-side Riemann-Liouville Fractional Derivative Operator $D_{0^{+}}^{\vartheta}$ of the jgeneralized p - k Mittag-Leffler Function is given by,

$$
\left(D_{0^{+}}^{\vartheta}\left[t^{\frac{\beta}{k}-1}{ }_{p}^{j} E_{k, \alpha, \beta}^{\gamma, q}\left(t^{\frac{\alpha}{k}}\right)\right]\right)(z)=p^{-\vartheta} z^{\frac{\beta}{k}-\vartheta-1}{ }_{p}^{j} E_{k, \alpha, \beta-k \vartheta}^{\gamma, q}\left(z^{\frac{\alpha}{k}}\right) .
$$

Where

$k, p \in R^{+}-\{0\} ; \alpha, \beta, \gamma \in C / k Z^{-} ; \operatorname{Re}(\alpha)>0, \operatorname{Re}(\beta)>0, \operatorname{Re}(\gamma)>0, j \in N_{0}, q \in(0,1) \cup N$ and $\operatorname{Re}(\vartheta)>0$.

Proof: Consider left hand side,

$$
A \equiv\left(D_{0^{+}}^{\vartheta}\left[t^{\frac{\beta}{k}-1}{ }_{p}^{j} E_{k, \alpha, \beta}^{\gamma, q}\left(t^{\frac{\alpha}{k}}\right)\right]\right)(z),
$$

by virtue of equation (1.22) and (2.1), we have

$$
A \equiv \frac{1}{\Gamma(1-\vartheta+[\operatorname{Re}(\vartheta)])}\left(\frac{d}{d z}\right)^{[\operatorname{Re}(\vartheta)]+1} \int_{0}^{z} \frac{t^{\frac{\beta}{k}-1}}{(z-t)^{\vartheta-\operatorname{Re}(\vartheta)}} \sum_{n=0}^{\infty} \frac{p(\gamma)_{(n+j) q, k}}{{ }_{p} \Gamma_{k}(n \alpha+\beta)} \frac{t^{\frac{n \alpha}{k}}}{(n+j) !} d t
$$

interchanging the order of integration and summation and evaluate the inner integral by substitute $t=z u$ and using the beta function formula, it gives

$$
A \equiv \frac{z^{\frac{\beta}{k}-\vartheta-1}}{\Gamma(\vartheta)} \sum_{n=0}^{\infty} \frac{p(\gamma)_{(n+j) q, k}}{{ }_{p} \Gamma_{k}(n \alpha+\beta)} \frac{z^{\frac{n \alpha}{k}}}{(n+j) !} \frac{\Gamma\left(\frac{n \alpha+\beta}{k}\right) \Gamma(\vartheta)}{\Gamma\left(\frac{n \alpha+\beta-k \vartheta}{k}\right)},
$$

using the equation (1.5), we have

$$
A \equiv p^{-\vartheta} z^{\frac{\beta}{k}-\vartheta-1}{ }_{p}^{j} E_{k, \alpha, \beta-k \vartheta}^{\gamma, q}\left(z^{\frac{\alpha}{k}}\right) .
$$

Hence, we get the desired result.

Theorem 3.4 The right-side Riemann-Liouville Fractional Derivative Operator $D_{-}^{\vartheta}$ of the jgeneralized p - k Mittag-Leffler Function is given by,

$$
\left(D_{-}^{\vartheta}\left[t^{-\frac{\beta}{k}+\vartheta}{ }_{p}^{j} E_{k, \alpha, \beta}^{\gamma, q}\left(t^{-\frac{\alpha}{k}}\right)\right]\right)(z)=p^{-\vartheta} z^{-\frac{\beta}{k}}{ }_{p}^{j} E_{k, \alpha, \beta-k \vartheta}^{\gamma, q}\left(z^{-\frac{\alpha}{k}}\right) .
$$

Where

$k, p \in R^{+}-\{0\} ; \alpha, \beta, \gamma \in C / k Z^{-} ; \operatorname{Re}(\alpha)>0, \operatorname{Re}(\beta)>0, \operatorname{Re}(\gamma)>0, j \in N_{0}, q \in(0,1) \cup N$ and $\operatorname{Re}(\vartheta)>0$. 
Proof: Consider left hand side,

$$
A \equiv\left(D_{-}^{\vartheta}\left[t^{-\frac{\beta}{k}+\vartheta}{ }_{p}^{j} E_{k, \alpha, \beta}^{\gamma, q}\left(t^{-\frac{\alpha}{k}}\right)\right]\right)(z),
$$

by virtue of equation (1.23) and (2.1), we have

$$
A \equiv \frac{1}{\Gamma(1-\vartheta+[\operatorname{Re}(\vartheta)])}\left(-\frac{d}{d z}\right)^{[\operatorname{Re}(\vartheta)]+1} \int_{z}^{\infty} \frac{z^{-\frac{\beta}{k}+\vartheta}}{(t-z)^{\vartheta-[R e(\vartheta)]}} \sum_{n=0}^{\infty} \frac{{ }_{p}(\gamma)_{(n+j) q, k}}{{ }_{p} \Gamma_{k}(n \alpha+\beta)} \frac{t^{-\frac{n \alpha}{k}}}{(n+j) !} d t
$$

interchanging the order of integration and summation and evaluate the inner integral by substitute $t=\frac{z}{u}$ and using the beta function formula, it gives

$$
A \equiv \frac{z^{-\frac{\beta}{k}}}{\Gamma(\vartheta)} \sum_{n=0}^{\infty} \frac{p(\gamma)_{(n+j) q, k}}{{ }_{p} \Gamma_{k}(n \alpha+\beta)} \frac{z^{-\frac{n \alpha}{k}}}{(n+j) !} \frac{\Gamma\left(\frac{n \alpha+\beta}{k}\right) \Gamma(\vartheta)}{\Gamma\left(\frac{n \alpha+\beta-k \vartheta}{k}\right)},
$$

using the equation (1.5), we have

$$
A \equiv p^{-\vartheta} z^{-\frac{\beta}{k}}{ }_{p}^{j} E_{k, \alpha, \beta-k \vartheta}^{\gamma, q}\left(z^{-\frac{\alpha}{k}}\right) .
$$

Hence, we get the desired result.

\section{Recurrence Relation and Integral Representation of the j- generalized p - k Mittag-Leffler Function}

In this section we evaluate the recurrence relations and integral representations of the j-generalized $\mathrm{p}$ - k Mittag-Leffler function.

Theorem 4.1 For $k, p \in R^{+}-\{0\} ; \alpha+r, \beta+s+k, \gamma \in C / k Z^{-} ; R(\alpha+r)>0, R(\beta+s+k)>$ $0, R(\gamma)>0, q \in(0,1) \cup N, j \in N_{0}$, we get

$$
\begin{gathered}
{ }_{p}^{j} E_{k, \alpha+r, \beta+s+k}^{\gamma, q}(z)-p{ }_{p}^{j} E_{k, \alpha+r, \beta+s+2 k}^{\gamma, q}(z)=\frac{p^{2}}{k^{2}}\left[(\alpha+r)^{2} z^{2}{ }_{p}^{j} \ddot{E}_{k, \alpha+r, \beta+s+3 k}^{\gamma, q}(z)\right. \\
+\left\{(\alpha+r)^{2}+(\alpha+r)(2 \beta+2 s+2 k) z\right\}{ }_{p}^{j} \dot{E}_{k, \alpha+r, \beta+s+3 k}^{\gamma, q}(z) \\
\left.+(\beta+s)(\beta+s+2 k){ }_{p}^{j} E_{k, \alpha+r, \beta+s+3 k}^{\gamma, q}(z)\right],
\end{gathered}
$$

where ${ }_{p}^{j} \dot{E}_{k, \alpha, \beta}^{\gamma, q}(z)=\frac{d}{d z}{ }_{p}^{j} E_{k, \alpha, \beta}^{\gamma, q}(z)$ and ${ }_{p}^{j} \ddot{E}_{k, \alpha, \beta}^{\gamma, q}(z)=\frac{d^{2}}{d z^{2}}{ }_{p}^{j} E_{k, \alpha, \beta}^{\gamma, q}(z)$.

Proof: The j-generalized p-k Mittag-Leffler function, from equation (2.1),

$$
{ }_{p}^{j} E_{k, \alpha+r, \beta+s+k}^{\gamma, q}(z)=\sum_{n=0}^{\infty} \frac{p(\gamma)_{(n+j) q, k} z^{n}}{{ }_{p} \Gamma_{k}(n(\alpha+r)+\beta+s+k)((n+j) !)},
$$

using equation (1.9), we have

$$
{ }_{p}^{j} E_{k, \alpha+r, \beta+s+k}^{\gamma, q}(z)=\sum_{n=0}^{\infty} \frac{k}{p_{p}} \frac{p(\gamma)_{(n+j) q, k} z^{n}}{\left.\Gamma_{k}(n(\alpha+r)+\beta+s)\{n(\alpha+r)+\beta+s)\right\}((n+j) !)},
$$

and

$$
{ }_{p}^{j} E_{k, \alpha+r, \beta+s+2 k}^{\gamma, q}(z)=\sum_{n=0}^{\infty} \frac{p(\gamma)_{(n+j) q, k} z^{n}}{{ }_{p} \Gamma_{k}(n(\alpha+r)+\beta+s+2 k)((n+j) !)},
$$


using equation (1.9), we have

$$
\begin{gathered}
{ }_{p}^{j} E_{k, \alpha+r, \beta+s+2 k}^{\gamma, q}(z)=\sum_{n=0}^{\infty} \frac{p(\gamma)_{(n+j) q, k} z^{n}}{{ }_{p} \Gamma_{k}(n(\alpha+r)+\beta+s)((n+j) !)} \\
\times \frac{k^{2}}{p^{2}} \frac{1}{\{n(\alpha+r)+\beta+s)\}\{n(\alpha+r)+\beta+s+k)\}}, \\
{ }_{p}^{j} E_{k, \alpha+r, \beta+s+2 k}^{\gamma, q}(z)=\sum_{n=0}^{\infty} \frac{k}{p^{2}}\left[\frac{1}{(n(\alpha+r)+\beta+s)}-\frac{1}{(n(\alpha+r)+\beta+s+k)}\right] \\
\times \frac{p(\gamma)(n+j) q, k}{{ }_{p} z_{k}(n(\alpha+r)+\beta+s)((n+j) !)}, \\
{ }_{p}^{j} E_{k, \alpha+r, \beta+s+2 k}^{\gamma, q}(z)=\frac{k}{p^{2}}\left[\frac{p}{k}{ }_{p}^{j} E_{k, \alpha+r, \beta+s+k}^{\gamma, q}(z)-S\right], \\
S=\frac{p}{k}{ }_{p}^{j} E_{k, \alpha+r, \beta+s+k}^{\gamma, q}(z)-\frac{p^{2}}{k}{ }_{p}^{j} E_{k, \alpha+r, \beta+s+2 k}^{\gamma, q}(z),
\end{gathered}
$$

where

$$
S=\sum_{n=0}^{\infty} \frac{p(\gamma)_{(n+j) q, k} z^{n}}{\left.{ }_{p} \Gamma_{k}(n(\alpha+r)+\beta+s)\{n(\alpha+r)+\beta+s+k)\right\}((n+j) !)},
$$

applying the simple identity $\frac{1}{u}=\frac{k}{u(u+k)}+\frac{1}{u+k}$; for $u=n(\alpha+r)+\beta+s+k$ to (4.5), we obtain,

$$
\begin{gathered}
S=\sum_{n=0}^{\infty} \frac{k_{p}(\gamma)_{(n+j) q, k} z^{n}}{{ }_{p} \Gamma_{k}(n(\alpha+r)+\beta+s)((n+j) !)} \times \frac{1}{\{n(\alpha+r)+\beta+s+k\}\{n(\alpha+r)+\beta+s+2 k\}} \\
+\sum_{n=0}^{\infty} \frac{p(\gamma)_{(n+j) q, k} z^{n}}{\left.{ }_{p} \Gamma_{k}(n(\alpha+r)+\beta+s)\{n(\alpha+r)+\beta+s+2 k)\right\}((n+j) !)}, \\
\quad S=\sum_{n=0}^{\infty} \frac{k\{n(\alpha+r)+\beta+s\}_{p}(\gamma)_{(n+j) q, k} z^{n}}{{ }_{p} \Gamma_{k}(n(\alpha+r)+\beta+s)((n+j) !)} \\
\quad \frac{1}{\{n(\alpha+r)+\beta+s\}\{n(\alpha+r)+\beta+s+k\}\{n(\alpha+r)+\beta+s+2 k\}} \\
\quad \times \frac{\sum_{n=0}^{\infty} \frac{\{n(\alpha+r)+\beta+s\}\{n(\alpha+r)+\beta+s+k\}_{p}(\gamma)_{(n+j) q, k} z^{n}}{\{n(\alpha+r)+\beta+s\}\{n(\alpha+r)+\beta+s+k\}\{n(\alpha+r)+\beta+s+2 k\}},}{p(n+\alpha+r)+\beta+s)((n+j) !)}
\end{gathered}
$$

using equation (1.9), we obtain

$$
\begin{gathered}
S=\sum_{n=0}^{\infty} \frac{k\{n(\alpha+r)+\beta+s\}_{p}(\gamma)_{(n+j) q, k} z^{n}}{\frac{k^{3}}{p^{3}} \Gamma_{k}(n(\alpha+r)+\beta+s+3 k)((n+j) !)} \\
+\sum_{n=0}^{\infty} \frac{\{n(\alpha+r)+\beta+s\}\{n(\alpha+r)+\beta+s+k\}_{p}(\gamma)_{(n+j) q, k} z^{n}}{\frac{k^{3}}{p^{3}} \Gamma_{k}(n(\alpha+r)+\beta+s+3 k)((n+j) !)} \\
S \frac{k^{3}}{p^{3}}=\sum_{n=0}^{\infty} \frac{p(\gamma)_{(n+j) q, k} z^{n}}{{ }_{p} \Gamma_{k}(n(\alpha+p)+\beta+s+3 k)((n+j) !)} \\
\times\left[n^{2}(\alpha+r)^{2}+2 n(\alpha+r)(\beta+s+k)+(\beta+s)(\beta+s+2 k)\right] .
\end{gathered}
$$


We now express each summation in the right hand side of (4.6) as follows:

$$
\begin{gathered}
\frac{d}{d z}\left[z_{p}^{j} E_{k, \alpha+r, \beta+s+3 k}^{\gamma, q}(z)\right]=\sum_{n=0}^{\infty} \frac{(n+1)_{p}(\gamma)_{(n+j) q, k} z^{n}}{{ }_{p} \Gamma_{k}(n(\alpha+r)+\beta+s+3 k)((n+j) !)} \\
z_{p}^{j} \dot{E}_{k, \alpha+r, \beta+s+3 k}^{\gamma, q}(z)+{ }_{p}^{j} E_{k, \alpha+r, \beta+s+3 k}^{\gamma, q}(z)=\sum_{n=0}^{\infty} \frac{(n+1)_{p}(\gamma)_{(n+j) q, k} z^{n}}{{ }_{p} \Gamma_{k}(n(\alpha+r)+\beta+s+3 k)((n+j) !)}, \\
z_{p}^{j} \dot{E}_{k, \alpha+r, \beta+s+3 k}^{\gamma, q}(z)=\sum_{n=0}^{\infty} \frac{n_{p}(\gamma)_{(n+j) q, k} z^{n}}{{ }_{p} \Gamma_{k}(n(\alpha+r)+\beta+s+3 k)((n+j) !)} .
\end{gathered}
$$

Again

$$
\frac{d^{2}}{d z^{2}}\left[z^{2}{ }_{p}^{j} E_{k, \alpha+r, \beta+s+3 k}^{\gamma, q}(z)\right]=\sum_{n=0}^{\infty} \frac{(n+1)(n+2)_{p}(\gamma)_{(n+j) q, k} z^{n}}{{ }_{p} \Gamma_{k}(n(\alpha+r)+\beta+s+3 k)((n+j) !)}
$$

and

$$
\begin{gathered}
\frac{d^{2}}{d z^{2}}\left[z^{2}{ }_{p}^{j} E_{k, \alpha+r, \beta+s+3 k}^{\gamma, q}(z)\right] \\
=z^{2}{ }_{p}^{j} \ddot{E}_{k, \alpha+r, \beta+s+3 k}^{\gamma, q}(z)+4 z_{p}^{j} \dot{E}_{k, \alpha+r, \beta+s+3 k}^{\gamma, q}(z)+2{ }_{p}^{j} E_{k, \alpha+r, \beta+s+3 k}^{\gamma, q}(z),
\end{gathered}
$$

from equation (4.8) and (4.9) we have

$$
\begin{gathered}
\sum_{n=0}^{\infty} \frac{\left\{n^{2}\right\}_{p}(\gamma)_{(n+j) q, k} z^{n}}{{ }_{p}(n(\alpha+r)+\beta+s+3 k)((n+j) !)}=z^{2}{ }_{p}^{j} \ddot{E}_{k, \alpha+r, \beta+s+3 k}^{\gamma, q}(z) \\
+4 z_{p}^{j} \dot{E}_{k, \alpha+r, \beta+s+3 k}^{\gamma, q}(z)-3 \sum_{n=0}^{\infty} \frac{\{n\}_{p}(\gamma)_{(n+j) q, k} z^{n}}{\Gamma_{k}(n(\alpha+r)+\beta+s+3 k)((n+j) !)},
\end{gathered}
$$

using equation (4.7), we have

$$
\begin{aligned}
& \sum_{n=0}^{\infty} \frac{\left\{n^{2}\right\}_{p}(\gamma)_{(n+j) q, k} z^{n}}{{ }_{p} \Gamma_{k}(n(\alpha+r)+\beta+s+3 k)((n+j) !)} \\
= & z^{2}{ }_{p}^{j} \ddot{E}_{k, \alpha+r, \beta+s+3 k}^{\gamma, q}(z)+z{ }_{p}^{j} \dot{E}_{k, \alpha+r, \beta+s+3 k}^{\gamma, q}(z),
\end{aligned}
$$

applying equation (4.4), (4.7) and (4.10) to (4.6), we get

$$
\begin{aligned}
\frac{k^{3}}{p^{3}} S= & (\alpha+r)^{2} z^{2}{ }_{p}^{j} \ddot{E}_{k, \alpha+r, \beta+s+3 k}^{\gamma, q}(z)+\left[(\alpha+r)^{2}+(\alpha+r)(2 \beta+2 s+2 k)\right] z \\
& \times{ }_{p}^{j} \dot{E}_{k, \alpha+r, \beta+s+3 k}^{\gamma, q}(z)+(\beta+s)(\beta+s+2 k){ }_{p}^{j} E_{k, \alpha+r, \beta+s+3 k}^{\gamma, q}(z),
\end{aligned}
$$

Hence.

Theorem 4.2 For $k, p \in R^{+}-\{0\} ; \alpha+r, \beta+s+k, \gamma \in C / k Z^{-} ; R(\alpha+r)>0, R(\beta+s+k)>$ $0, R(\gamma)>0, q \in(0,1) \cup N, j \in N_{0}$, we get

$$
\int_{0}^{1} t^{\beta+s+k-1}{ }_{p}^{j} E_{k, \alpha+r, \beta+s}^{\gamma, q}\left(t^{\alpha+r}\right) d t=\frac{p}{k}{ }_{p}^{j} E_{k, \alpha+r, \beta+s+k}^{\gamma, q}(1)-\frac{p^{2}}{k}{ }_{p}^{j} E_{k, \alpha+r, \beta+s+2 k}^{\gamma, q}(1) .
$$

Proof: Put $z=1$ in equation (4.4) and (4.5), we have

$$
\begin{gathered}
S=\frac{p}{k}{ }_{p}^{j} E_{k, \alpha+r, \beta+s+k}^{\gamma, q}(1)-\frac{p^{2}}{k}{ }_{p}^{j} E_{k, \alpha+r, \beta+s+2 k}^{\gamma, q}(1) \\
=\sum_{n=0}^{\infty} \frac{p(\gamma)_{(n+j) q, k}}{\left.{ }_{p} \Gamma_{k}(n(\alpha+r)+\beta+s)\{n(\alpha+r)+\beta+s+k)\right\}((n+j) !)},
\end{gathered}
$$


now consider the integral,

$$
A \equiv \int_{0}^{1} t^{\beta+s+k-1}{ }_{p}^{j} E_{k, \alpha+r, \beta+s}^{\gamma, q}\left(t^{\alpha+r}\right) d t
$$

using the equation (2.1), we have

$$
\begin{aligned}
A & \equiv \sum_{n=0}^{\infty} \frac{p(\gamma)_{(n+j) q, k}}{{ }_{p} \Gamma_{k}(n(\alpha+r)+\beta+s)((n+j) !)} \int_{0}^{1} t^{n(\alpha+r)+\beta+s+k-1} d t, \\
A & \equiv \sum_{n=0}^{\infty} \frac{p(\gamma)_{(n+j) q, k}}{\left.{ }_{p} \Gamma_{k}(n(\alpha+r)+\beta+s)\{n(\alpha+r)+\beta+s+k)\right\}((n+j) !)},
\end{aligned}
$$

from equation (4.12), we have the desired result.

Theorem 4.3 For $k, p \in R^{+}-\{0\} ; \alpha, \beta, \gamma, \delta \in C ; R(\alpha)>0, R(\beta)>0, R(\gamma)>0, R(\delta)>$ 0 and $q \in(0,1) \cup N, j \in N_{0}$ then

$$
p_{p}^{\delta}{ }_{p}^{j} E_{k, \alpha, \beta+\delta k}^{\gamma, q}(z)=\frac{1}{\Gamma(\delta)} \int_{0}^{1} u^{\frac{\beta}{k}-1}(1-u)^{\delta-1}{ }_{p}^{j} E_{k, \alpha, \beta}^{\gamma, q}\left(z u^{\frac{\alpha}{k}}\right) d u .
$$

Proof : Consider the right side integral and using equation (2.1), we have

$$
\begin{gathered}
A \equiv \frac{1}{\Gamma(\delta)} \int_{0}^{1} u^{\frac{\beta}{k}-1}(1-u)^{\delta-1}{ }_{p} E_{k, \alpha, \beta}^{\gamma, q}\left(z u^{\frac{\alpha}{k}}\right) d u \\
A \equiv \frac{1}{\Gamma(\delta)} \sum_{n=0}^{\infty} \frac{p(\gamma)_{(n+j) q, k} z^{n}}{{ }_{p}(n \alpha+\beta)((n+j) !)} \int_{0}^{1} u^{\frac{\alpha n+\beta}{k}-1}(1-u)^{\delta-1} d u
\end{gathered}
$$

using the definition of Beta function, we have

$$
A \equiv \frac{1}{\Gamma(\delta)} \sum_{n=0}^{\infty} \frac{p(\gamma)_{(n+j) q, k} z^{n}}{{ }_{p} \Gamma_{k}(n \alpha+\beta)((n+j) !)} \frac{\Gamma\left(\frac{\alpha n+\beta}{k}\right) \Gamma(\delta)}{\Gamma\left(\frac{\alpha n+\beta}{k}+\delta\right)},
$$

applying equation (1.5), we have

$$
A \equiv \sum_{n=0}^{\infty} \frac{p^{\delta}{ }_{p}(\gamma)_{(n+j) q, k} z^{n}}{{ }_{p} \Gamma_{k}(n \alpha+\beta+\delta k)((n+j) !)}=p^{\delta}{ }_{p}^{j} E_{k, \alpha, \beta+\delta k}^{\gamma, q}(z),
$$

Hence.

Theorem 4.4 For $k, p \in R^{+}-\{0\} ; \beta, \gamma \in C ; R(\beta)>0, R(\gamma)>0$ and $\alpha, q \in N, j \in N_{0}$, then

$$
{ }_{p}^{j} E_{k, k \alpha, \beta}^{\gamma, q}(z)=\frac{p(\gamma)_{j q, k}}{{ }_{p} \Gamma_{k}(\beta)} \prod_{i=1}^{q} \prod_{l=1}^{\alpha} \frac{\Gamma\left(b_{l}\right)}{\Gamma\left(a_{i}\right) \Gamma\left(b_{l}-a_{i}\right)} \int_{0}^{1} u^{a_{i}-1}(1-u)^{b_{l}-a_{i}-1} E_{1, j+1}\left(u z \frac{p^{q-\alpha} q^{q}}{\alpha^{\alpha}}\right) d u .
$$

Where $a_{i}=\frac{\frac{\gamma}{k}+q j+i-1}{q}$ and $b_{l}=\frac{\frac{\beta}{k}+l-1}{\alpha}$.

Proof : Using definition of j-generalized p-k Mittag- Leffler function, from equation (2.1),

$$
A \equiv{ }_{p}^{j} E_{k, k \alpha, \beta}^{\gamma, q}(z)=\sum_{n=0}^{\infty} \frac{p(\gamma)_{(n+j) q, k} z^{n}}{{ }_{p} \Gamma_{k}(n k \alpha+\beta)((n+j) !)},
$$


using relation (1.11),we have

$$
A \equiv \sum_{n=0}^{\infty} \frac{p(\gamma)_{j q, k p}(\gamma+j q k)_{n q, k} z^{n}}{p(\beta)_{n \alpha, k p} \Gamma_{k}(\beta)((n+j) !)}=\sum_{n=0}^{\infty} D \frac{p(\gamma)_{j q, k} z^{n}}{{ }_{p} \Gamma_{k}(\beta)((n+j) !)} .
$$

Where $D \equiv \frac{p(\gamma+j q k)_{n q, k}}{p(\beta)_{n \alpha, k}}$,

using equation (1.6), we have

$$
D \equiv \frac{{ }_{p}(\gamma+j q k)_{n q, k}}{p(\beta)_{n \alpha, k}}=\frac{p^{n(q-\alpha)}\left(\frac{\gamma+j q k}{k}\right)_{q n}}{\left(\frac{\beta}{k}\right)_{n \alpha}}
$$

using the relation given by equation (1.7), we have

$$
\begin{gathered}
D \equiv \frac{p^{(q-\alpha) n} q^{q n} \prod_{i=1}^{q}\left(\frac{\frac{\gamma}{k}+j q+i-1}{q}\right)_{n}}{\alpha^{\alpha n} \prod_{l=1}^{\alpha}\left(\frac{\frac{\beta}{k}+l-1}{\alpha}\right)_{n}}, \\
\text { let } a_{i}=\frac{\frac{\gamma}{k}+j q+i-1}{q} \text { and } b_{l}=\frac{\frac{\beta}{k}+l-1}{\alpha}, \\
D \equiv\left(\frac{p^{(q-\alpha)} q^{q}}{\alpha^{\alpha}}\right)^{n} \prod_{i=1}^{q} \prod_{l=1}^{\alpha} \frac{\left(a_{i}\right)_{n}}{\left(b_{l}\right)_{n}}, \\
D \equiv\left(\frac{p^{(q-\alpha)} q^{q}}{\alpha^{\alpha}}\right)^{n} \prod_{i=1}^{q} \prod_{l=1}^{\alpha} \frac{\Gamma\left(a_{i}+n\right) \Gamma\left(b_{l}\right)}{\Gamma\left(b_{l}+n\right) \Gamma\left(a_{i}\right)}, \\
D \equiv\left(\frac{p^{(q-\alpha)} q^{q}}{\alpha^{\alpha}}\right)^{n} \prod_{i=1}^{q} \prod_{l=1}^{\alpha} \frac{\Gamma\left(b_{l}\right)}{\Gamma\left(b_{l}-a_{i}\right) \Gamma\left(a_{i}\right)} \frac{\Gamma\left(a_{i}+n\right) \Gamma\left(b_{l}-a_{i}\right)}{\Gamma\left(b_{l}-a_{i}+a_{i}+n\right)},
\end{gathered}
$$

using the definition of Beta function, we have

$$
D \equiv\left(\frac{p^{(q-\alpha)} q^{q}}{\alpha^{\alpha}}\right)^{n} \prod_{i=1}^{q} \prod_{l=1}^{\alpha} \frac{\Gamma\left(b_{l}\right)}{\Gamma\left(b_{l}-a_{i}\right) \Gamma\left(a_{i}\right)} \int_{0}^{1} u^{a_{i}+n-1}(1-u)^{b_{l}-a_{i}-1} d u,
$$

from equation (4.15) and (4.16), we have

$$
A \equiv \frac{p(\gamma)_{j q, k}}{{ }_{p} \Gamma_{k}(\beta)} \prod_{i=1}^{q} \prod_{l=1}^{\alpha} \frac{\Gamma\left(b_{l}\right)}{\Gamma\left(b_{j}-a_{i}\right) \Gamma\left(a_{i}\right)} \int_{0}^{1} u^{a_{i}-1}(1-u)^{b_{j}-a_{i}-1} \sum_{n=0}^{\infty} \frac{(u z)^{n}}{(n+j) !}\left(\frac{p^{(q-\alpha)} q^{q}}{\alpha^{\alpha}}\right)^{n} d u
$$

Hence.

Theorem 4.5 For $k, p \in R^{+}-\{0\} ; \alpha, \beta, \gamma \in C ; R(\alpha)>0, R(\beta)>0, R(\gamma)>0$ and $q \in$ $(0,1) \cup N, j \in N_{0}$ then

$$
{ }_{p}^{j} E_{k, \alpha, \beta}^{\gamma, q}(z)=\frac{p^{j q}}{\Gamma\left(\frac{\gamma}{k}\right)} \int_{0}^{\infty} e^{-t} t^{\left(\frac{\gamma}{k}+j q-1\right)}{ }_{p}^{j} E_{k, \alpha, \beta}^{1,0}\left(z t^{q} p^{q}\right) d t .
$$

Proof: Using definition of j-generalized p-k Mittag-Leffler function, equation (2.1), we have

$$
{ }_{p}^{j} E_{k, \alpha, \beta}^{\gamma, q}(z)=\sum_{n=0}^{\infty} \frac{p(\gamma)_{(n+j) q, k} z^{n}}{{ }_{p} \Gamma_{k}(n \alpha+\beta)((n+j) !)},
$$

using equation (1.5) and (1.6), we have

$$
{ }_{p}^{j} E_{k, \alpha, \beta}^{\gamma, q}(z)=\sum_{n=0}^{\infty} \frac{z^{n}}{{ }_{p} \Gamma_{k}(n \alpha+\beta)((n+j) !)} \frac{p^{(n+j) q} \Gamma\left(\frac{\gamma}{k}+q(n+j)\right)}{\Gamma\left(\frac{\gamma}{k}\right)},
$$




$$
\begin{gathered}
{ }_{p}^{j} E_{k, \alpha, \beta}^{\gamma, q}(z)=\sum_{n=0}^{\infty} \frac{z^{n}}{{ }_{p} \Gamma_{k}(n \alpha+\beta)((n+j) !)} \frac{p^{q(n+j)}}{\Gamma\left(\frac{\gamma}{k}\right)} \int_{0}^{\infty} e^{-t} t^{\left(\frac{\gamma}{k}+q(n+j)-1\right)} d t \\
{ }_{p}^{j} E_{k, \alpha, \beta}^{\gamma, q}(z)=\frac{p^{j q}}{\Gamma\left(\frac{\gamma}{k}\right)} \int_{0}^{\infty} e^{-t} t^{\left(\frac{\gamma}{k}+j q-1\right)} \sum_{n=0}^{\infty} \frac{z^{n} p^{q n} t^{q n}}{{ }_{p} \Gamma_{k}(n \alpha+\beta)((n+j) !)} d t \\
{ }_{p}^{j} E_{k, \alpha, \beta}^{\gamma, q}(z)=\frac{p^{j q}}{\Gamma\left(\frac{\gamma}{k}\right)} \int_{0}^{\infty} e^{-t} t^{\left(\frac{\gamma}{k}+j q-1\right)}{ }_{p}^{j} E_{k, \alpha, \beta}^{1,0}\left(z t^{q} p^{q}\right) d t .
\end{gathered}
$$

Hence.

\section{Integral Transform of the j-generalized P-K Mittag-Leffler Function}

In this section we evaluate Mellin-Barnes integral representation of j-generalized p-k MittagLeffler function, relationship with Fox H-function and Wright hypergeometric function. Also evaluate Euler Beta Transform, Laplace Transform and Mellin Transform of j-generalized p-k Mittag-Leffler function.

Mellin-Barnes integral representation of the j-generalized p-k Mittag-Leffler function.

Theorem 5.1 Let $k, p \in R^{+}-\{0\} ; \operatorname{Re}(\alpha)>0, \operatorname{Re}(\beta)>0, \operatorname{Re}(\gamma)>0$, and $q \in(0,1) \cup N, j \in N_{0}$, then the j-generalized p-k Mittag-Leffler function is represented by the Mellin-Barnes integral as,

$$
{ }_{p}^{j} E_{k, \alpha, \beta}^{\gamma, q}(z)=\frac{k p^{j q-\frac{\beta}{k}}}{2 \pi i \Gamma\left(\frac{\gamma}{k}\right)} \int_{L} \frac{\Gamma(s) \Gamma(1-s) \Gamma\left(\frac{\gamma}{k}+j q-q s\right)}{\Gamma\left(\frac{\beta}{k}-\frac{\alpha s}{k}\right) \Gamma(1+j-s)}\left(-z p^{q-\frac{\alpha}{k}}\right)^{-s} d s .
$$

Where $|\arg z|<\pi$; the contour integration beginning at $-i \infty$ and ending at $+i \infty$, and indented to separate the poles of the integrand as $s=-n$ for every $n \in N_{0}$ (to the left) from those at $s=\frac{\frac{\gamma}{k}+n}{q}$ for every $n \in N_{0}$ (to the right).

Proof Consider the integral on right side of equation(5.1) and use the theorem of calculus of residues,

$$
A \equiv \frac{k p^{j q-\frac{\beta}{k}}}{2 \pi i \Gamma\left(\frac{\gamma}{k}\right)} \int_{L} \frac{\Gamma(s) \Gamma(1-s) \Gamma\left(\frac{\gamma}{k}+j q-q s\right)}{\Gamma\left(\frac{\beta}{k}-\frac{\alpha s}{k}\right) \Gamma(1+j-s)}\left(-z p^{q-\frac{\alpha}{k}}\right)^{-s} d s
$$

$=2 \pi i[$ sum of the residues at the poles $s=0,-1,-2, \ldots]$

$$
\begin{gathered}
A \equiv \frac{k p^{j q-\frac{\beta}{k}}}{\Gamma\left(\frac{\gamma}{k}\right)} \sum_{n=0}^{\infty} \operatorname{Re}_{s=-n}(s+n)\left[\frac{\Gamma(s) \Gamma(1-s) \Gamma\left(\frac{\gamma}{k}+j q-q s\right)}{\Gamma\left(\frac{\beta}{k}-\frac{\alpha s}{k}\right) \Gamma(1+j-s)}\right]\left(-z p^{q-\frac{\alpha}{k}}\right)^{-s} \\
A \equiv \frac{k p^{j q-\frac{\beta}{k}}}{\Gamma\left(\frac{\gamma}{k}\right)} \sum_{n=0}^{\infty} \lim _{s \rightarrow-n}\left[\frac{\pi(s+n)}{\sin \pi s} \frac{\Gamma\left(\frac{\gamma}{k}+j q-q s\right)}{\Gamma(1+j-s) \Gamma\left(\frac{\beta}{k}-\frac{\alpha s}{k}\right)}\right]\left(-z p^{q-\frac{\alpha}{k}}\right)^{-s} \\
A \equiv \frac{k p^{j q-\frac{\beta}{k}}}{\Gamma\left(\frac{\gamma}{k}\right)} \sum_{n=0}^{\infty}\left[\frac{\Gamma\left(\frac{\gamma}{k}+j q+q n\right)}{\Gamma(1+j+s) \Gamma\left(\frac{\beta}{k}+\frac{\alpha n}{k}\right)}\right]\left(z p^{q-\frac{\alpha}{k}}\right)^{n}
\end{gathered}
$$

using equations (1.5) and (1.6), we have,

$$
A \equiv{ }_{p}^{j} E_{k, \alpha, \beta}^{\gamma, q}(z) .
$$

Hence.

Theorem 5.2 Let $k, p \in R^{+}-\{0\} ; \operatorname{Re}(\alpha)>0, \operatorname{Re}(\beta)>0, \operatorname{Re}(\gamma)>0$, and $q \in(0,1) \cup N, j \in N_{0}$ 
then the j-generalized p-k Mittag-Leffler function is given in the form of Fox H-function, as.

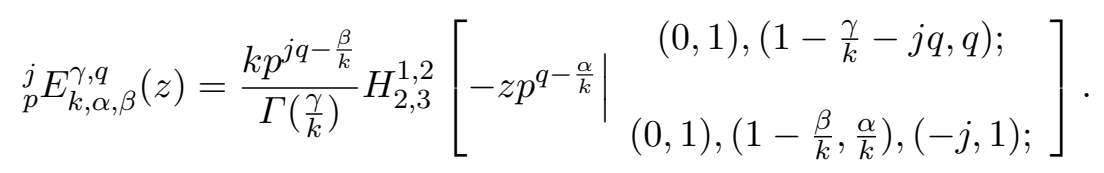

Proof. Using the equations (5.1) and (1.25), we get the desired result.

\section{Relationship with Wright hypergeometric function}

Theorem 5.3 Let $k, p \in R^{+}-\{0\} ; \operatorname{Re}(\alpha)>0, \operatorname{Re}(\beta)>0, \operatorname{Re}(\gamma)>0$, and $q \in(0,1) \cup N, j \in N_{0}$, then the j-generalized p-k Mittag-Leffler function is given in the form of Wright hypergeometric function, as.

$$
{ }_{p}^{j} E_{k, \alpha, \beta}^{\gamma, q}(z)=\frac{k p^{j q-\frac{\beta}{k}}}{\Gamma\left(\frac{\gamma}{k}\right)}{ }_{2} \Psi_{2}\left[\begin{array}{c}
(1,1)\left(\frac{\gamma}{k}+j q, q\right) ; \\
z p^{q-\frac{\alpha}{k}} \\
\left(\frac{\beta}{k}, \frac{\alpha}{k}\right)(1+j, 1) ;
\end{array}\right] .
$$

Proof. Using the equations (5.1) and (1.24), we get the desired result.

Euler Beta Transform, Laplace Transform and Mellin Transform of j-generalized p-k Mittag-Leffler function

Theorem 5.4 Let $k, p \in R^{+}-\{0\} ; a, b, \sigma \in C ; \operatorname{Re}(\alpha)>0, \operatorname{Re}(\beta)>0, \operatorname{Re}(\gamma)>0, \operatorname{Re}(\sigma)>0$ and $q \in(0,1) \cup N, j \in N_{0}$, then Euler Beta Transform of j-generalized p-k Mittag-Leffler function, is given by,

$$
\int_{0}^{1} z^{a-1}(1-z)^{b-1}{ }_{p}^{j} E_{k, \alpha, \beta}^{\gamma, q}\left(x z^{\sigma}\right) d z=\frac{k p^{j q-\frac{\beta}{k}} \Gamma(b)}{\Gamma\left(\frac{\gamma}{k}\right)}{ }_{3} \psi_{3}\left[\begin{array}{c}
(1,1),\left(\frac{\gamma}{k}+q j, q\right),(a, \sigma) ; \\
x p^{q-\frac{\alpha}{k}} \\
\left(\frac{\beta}{k}, \frac{\alpha}{k}\right),(1+j, 1),(a+b, \sigma) ;
\end{array}\right]
$$

Proof Consider the left side integral and using equation (5.4), we have

$$
\begin{gathered}
A \equiv \int_{0}^{1} z^{a-1}(1-z)^{b-1}{ }_{p}^{j} E_{k, \alpha, \beta}^{\gamma, q}\left(x z^{\sigma}\right) d z \\
A \equiv \sum_{n=0}^{\infty} \frac{p(\gamma)_{(n+j) q, k} x^{n}}{{ }_{p}(n \alpha+\beta)(n+j) !} \int_{0}^{1} z^{\sigma n+a-1}(1-z)^{b-1} d z,
\end{gathered}
$$

using definition of Beta function, we have

$$
A \equiv \sum_{n=0}^{\infty} \frac{p(\gamma)_{(n+j) q, k} x^{n}}{{ }_{p} \Gamma_{k}(n \alpha+\beta)(n+j) !} B(\sigma n+a, b)
$$

using equation (1.5),(1.6) and (1.24), we have

$$
A \equiv \frac{k p^{j q-\frac{\beta}{k}} \Gamma(b)}{\Gamma\left(\frac{\gamma}{k}\right)}{ }_{3} \psi_{3}\left[\begin{array}{c}
(1,1),\left(\frac{\gamma}{k}+q j, q\right),(a, \sigma) \\
x p^{q-\frac{\alpha}{k}} \\
\left(\frac{\beta}{k}, \frac{\alpha}{k}\right),(1+j, 1),(a+b, \sigma) ;
\end{array}\right] .
$$

Hence.

Theorem 5.5 The Laplace transform of j-generalizedp-k Mittag-Leffler function, is given by,

$$
\int_{0}^{\infty} z^{a-1} e^{-z s j} E_{k, \alpha, \beta}^{\gamma, q}\left(x z^{\sigma}\right) d z=\frac{k p^{j q-\frac{\beta}{k}} s^{-a}}{\Gamma\left(\frac{\gamma}{k}\right)}{ }_{3} \psi_{2}\left[\begin{array}{c}
(1,1),\left(\frac{\gamma}{k}+q j, q\right),(a, \sigma) ; \\
\frac{x p^{q-\frac{\alpha}{k}}}{s^{\sigma}} \\
\left(\frac{\beta}{k}, \frac{\alpha}{k}\right)(1+j, 1) ;
\end{array}\right]
$$


Where $k, p \in R^{+}-\{0\} ; a, \sigma \in C ; \operatorname{Re}(\alpha)>0, \operatorname{Re}(\beta)>0, \operatorname{Re}(\gamma)>0, \operatorname{Re}(\sigma)>0$ and $q \in$ $(0,1) \cup N, j \in N_{0}$, and $\left|\frac{x}{s^{\sigma}}\right|<1$.

Proof Consider the right side integral and using equation(2.1), we have

$$
\begin{gathered}
A \equiv \int_{0}^{\infty} z^{a-1} e_{p}^{-z s j} E_{k, \alpha, \beta}^{\gamma, q}\left(x z^{\sigma}\right) d z \\
A \equiv \sum_{n=0}^{\infty} \frac{p(\gamma)_{(n+j) q, k}}{{ }_{p} \Gamma_{k}(n \alpha+\beta)} \frac{x^{n}}{(n+j) !} \int_{0}^{\infty} z^{n \sigma+a-1} e^{-z s} d z,
\end{gathered}
$$

using definition of gamma function, we have

$$
A \equiv s^{-a} \quad \sum_{n=0}^{\infty} \frac{p(\gamma)_{(n+j) q, k}}{{ }_{p} \Gamma_{k}(n \alpha+\beta)(n+j) !} \Gamma(\sigma n+a)\left(\frac{x}{s^{\sigma}}\right)^{n}
$$

using equation (1.5),(1.6) and (1.24), we have

$$
A \equiv \frac{k p^{j q-\frac{\beta}{k}} s^{-a}}{\Gamma\left(\frac{\gamma}{k}\right)}{ }_{3} \psi_{2}\left[\begin{array}{r}
(1,1),\left(\frac{\gamma}{k}+q j, q\right),(a, \sigma) ; \\
\frac{x p^{q-\frac{\alpha}{k}}}{s^{\sigma}} \\
\left(\frac{\beta}{k}, \frac{\alpha}{k}\right)(1+j, 1) ;
\end{array}\right] .
$$

Hence.

Theorem 5.6 The Mellin transform of j-generalized p-k Mittag-Leffler function, is given by,

$$
\int_{0}^{\infty} t^{s-1}{ }_{p}^{j} E_{k, \alpha, \beta}^{\gamma, q}(-w t) d t=\frac{k p^{q j-\frac{\beta}{k}} \Gamma(s) \Gamma(1-s) \Gamma\left(\frac{\gamma}{k}+q j-q s\right)}{\Gamma\left(\frac{\beta}{k}-\frac{\alpha s}{k}\right) \Gamma\left(\frac{\gamma}{k}\right) \Gamma(1+j-s)}\left(\frac{p^{\frac{\alpha}{k}-q}}{w}\right)^{s}
$$

Where $k, p \in R^{+}-\{0\} ; a, \sigma \in C ; \operatorname{Re}(\alpha)>0, \operatorname{Re}(\beta)>0, \operatorname{Re}(\gamma)>0, \operatorname{Re}(s)>0$ and $q \in$ $(0,1) \cup N, j \in N_{0}$.

Proof Putting $\mathrm{z}=-\mathrm{wt}$ in equation (5.1), we have

$$
\begin{gathered}
{ }_{p}^{j} E_{k, \alpha, \beta}^{\gamma, q}(-w t)=\frac{k p^{j q-\frac{\beta}{k}}}{2 \pi i \Gamma\left(\frac{\gamma}{k}\right)} \int_{L} \frac{\Gamma(s) \Gamma(1-s) \Gamma\left(\frac{\gamma}{k}+j q-q s\right)}{\Gamma\left(\frac{\beta}{k}-\frac{\alpha s}{k}\right) \Gamma(1+j-s)}\left(-w t p^{q-\frac{\alpha}{k}}\right)^{-s} d s . \\
{ }_{p}^{j} E_{k, \alpha, \beta}^{\gamma, q}(-w t)=\frac{k p^{j q-\frac{\beta}{k}}}{2 \pi i \Gamma\left(\frac{\gamma}{k}\right)} \int_{L} f^{*}(s)(t)^{-s} d s .
\end{gathered}
$$

where

$$
f^{*}(s)=\frac{\Gamma(s) \Gamma(1-s) \Gamma\left(\frac{\gamma}{k}+j q-q s\right)}{\Gamma\left(\frac{\gamma}{k}\right) \Gamma\left(\frac{\beta}{k}-\frac{\alpha s}{k}\right) \Gamma(1+j-s)}\left(-w p^{q-\frac{\alpha}{k}}\right)^{-s}
$$

using equation (1.28),(1.29) and (5.7), which immediately leads to (3.9).

Particular cases:Putting some particular values of $j, p, q, k, \alpha, \beta, \gamma$ we obtain all the results given by [1], [3],[8],[9],[10],[11],,[12],[13] and [14].

\section{References}

[1] A. K. Shukla and J.C. Prajapati. On the generalization of Mittag-Leffler function and its properties. Journal of Mathematical Analysis and Applications,336 (2007) 797-811.

[2] A. Wiman. Uber den fundamental Satz in der Theories der Funktionen $E_{\alpha}(z)$, Acta Math. 29 (1905) 191-201.

[3] G.A. Dorrego and R.A. Cerutti. The K-Mittag-Leffler Function. Int. J.Contemp. Math. Sciences, Vol. 7 (2012) No. 15, 705-716. 
[4] G. Mittag-Leffler. Sur la nouvellefonction $E_{\alpha}(z)$ C.RAcad. Sci. Paris 137(1903) 554-558.

[5] H. Kilbas, H. Srivastava, J. Trujillo, Theory and Application of Fractional Differential Equations, Elsevier, 2006.

[6] H.M. Srivastava, H.L. Manocha, A Treatise on Generating Functions, John Wiley and Sons / Horword, New York/ Chichester,1984.

[7] I.N. Sneddon, The Use of Integral Transforms, Tata McGraw-Hill, New Delhi,1979.

[8]Kuldeep Singh Gehlot, The Generalized K- Mittag-Leffler function. Int. J. Contemp. Math. Sciences, Vol. 7 (2012) No. 45, 2213-2219.

[9] Kuldeep Singh Gehlot, Two Parameter Gamma Function and it's Properties, arXiv:1701.01052v1[math.CA] 3 Jan 2017.

[10] Kuldeep Singh Gehlot, The p-k Mittag-Liffler function, Palestine Journal of Mathematics, Vol. $7(2)(2018), 628-632$.

[11] Kuldeep Singh Gehlot, Fractional Integral and Diff. of p-k Mittag-Leffler function, under publication.

[12] Kuldeep Singh Gehlot, Recurrence relation and Integral representation of p-k Mittag-Leffler function, under publication.

[13] kuldeep Singh Gehlot, CR Choudhary and Anita Punia, Integral Transform of p-k Mittag -Leffler function, JETIR September 2018, Volume 5, Issue 9, 722-730.

[14] Luciano Leonardo Luque, On a Generalized Mittag-Leffler Function, International Journal of Mathematical Analysis, Vol. 13, 2019, no. 5, 223 - 234.

[15] Rafael Diaz and Eddy Pariguan. On hypergeometric functions and Pochhammer k-symbol. Divulgaciones Mathematicas, Vol. 15 No. 2 (2007) 179-192.

[16] S.G. Samok, A.A. Kilbas, O.I. Marichev, Fractional Integrals and Derivatives,Theory and Applications. Gordon and Breach, New York, 1993.

[17] T. R. Prabhakar. A singular integral equation with a generalized Mittag-Leffler function in the kernel. Yokohama Math. J. 19 (1971), 7-15. 